\title{
Influência da Localização Geográfica no Acesso às Terapias de Reperfusão e Mortalidade de Pacientes com IAMcSST em Sergipe: Registro VICTIM
}

\author{
Influence of Geographical Location on Access to Reperfusion Therapies and Mortality of Patients with STEMI \\ in Sergipe: VICTIM Register
}

\author{
Jeferson Cunha Oliveira, ${ }^{1,2}$ Guilherme José dos Santos Ferreira, ${ }^{3}$ Jussiely Cunha Oliveira, ${ }^{3}$ Ticiane Clair \\ Remacre Munareto Lima, ${ }^{10}$ Ikaro Daniel de Carvalho Barreto, ${ }^{40}$ Laís Costa Souza Oliveira, ${ }^{1,2}$ Larissa Andreline \\ Maia Arcelino, ${ }^{1,5}$ Antônio Carlos Sousa, ${ }^{3}$ José Augusto Soares Barreto-Filho ${ }^{1,3,6}$ \\ Universidade Federal de Sergipe - Programa de Pós-Graduação em Ciências da Saúde da Universidade Federal de Sergipe, ${ }^{1}$ São Cristóvão, SE - Brasil \\ Hospital Primavera, ${ }^{2}$ Aracaju, SE - Brasil \\ Universidade Federal de Sergipe, ${ }^{3}$ São Cristóvão, SE - Brasil \\ Universidade Federal Rural de Pernambuco - Núcleo de Pós-graduação em Biometria e Estatística Aplicada, ${ }^{4}$ Recife, PE - Brasil \\ Hospital Universitário da Universidade Federal de Sergipe (HU-UFS), ${ }^{5}$ Aracaju, SE - Brasil \\ Fundação São Lucas - Centro de Ensino e Pesquisa, ${ }^{6}$ Aracaju, SE - Brasil
}

\section{Resumo}

Fundamento: A concentração de serviços de alta complexidade em Aracaju/SE pode proporcionar disparidade na qualidade assistencial para os pacientes do SUS com infarto agudo do miocárdio com supradesnivelamento do segmento ST (IAMcSST) cujos sintomas se iniciaram em outras regiões de saúde do estado.

Objetivo: Avaliar disparidades no acesso às terapias de reperfusão e mortalidade em 30 dias, entre pacientes com IAMcSST, usuários do SUS, em cada uma das 7 regiões de saúde em Sergipe.

Métodos: Foram avaliados 844 pacientes com IAMcSST no período de 2014 a 2018 atendidos pelo único hospital com capacidade de ofertar intervenção coronariana percutânea (ICP) primária para usuários do SUS no estado de Sergipe. Os pacientes foram divididos em 7 grupos de acordo com o local de início dos sintomas e obedecendo a divisão já existente das regiões de saúde do Estado. Para comparação entre grupos, foi considerada diferença significativa quando $\mathbf{p}<0,05$.

Resultados: Do total de 844 pacientes vítimas de IAMCSST e transferidos ao hospital com ICP que atende pacientes do SUS, 386 pacientes $(45,8 \%)$ realizaram angioplastia primária. A taxa média do uso de fibrinolítico foi de $2,6 \%$, não havendo diferenças entre as regiões. O tempo médio total de chegada ao hospital com ICP foi de $21 \mathrm{~h}^{2} 5^{\prime}$ com mediana de $10 \mathrm{~h} 22^{\prime}\left(6 \mathrm{~h} 30^{\prime}-22 \mathrm{~h} 52^{\prime}\right)$. A mortalidade total em 30 dias foi 12,8\%, mas sem diferenças entre as regiões, mesmo quando ajustada para idade e sexo.

Conclusões: Este estudo revela que os fibrinolíticos são subutilizados em todo o estado e que existe um atraso significativo no acesso ao hospital com ICP, em todas as regiões de saúde de Sergipe.

Palavras-chave: Doenças Cardiovasculares; Infarto do Miocárdio; Reperfusão Miocárdica; Mortalidade; Epidemiologia; Estudos de Corte Transversal.

\section{Abstract}

Background: The concentration of high-complexity services in Aracaju, Sergipe can impose certain disparity in the quality of care for the patients with ST-segment elevation acute myocardial infarction (STEMI) (STEMI) who receive care from Brazil's Unified Health System (SUS, acronym in Portuguese) and whose symptoms started in other health regions of the state.

Correspondência: Jeferson Cunha Oliveira

Universidade Federal de Sergipe - Programa de Pós-Graduação em Ciências da Saúde da Universidade Federal de Sergipe - Av. Marechal Rondon,s/n

CEP 49100-000, Jardim Rosa Elze, São Cristóvão, SE - Brasil

E-mal: jeferson-cunha@hotmail.com.br

Artigo recebido em 06/01/2020, revisado em 04/06/2020, aceito em 05/08/2020

DOI: https://doi.org/10.36660/abc.20200015 
Objective: To evaluate disparities in access to reperfusion therapies and 30-day mortality, among patients with STEMI, who were users of SUS, in each of the 7 health regions of Sergipe.

Methods: A total of 844 patients with STEMI in the period from 2014 to 2018, assisted by the only hospital with the capacity to offer primary percutaneous coronary intervention (PPCI) to SUS users in the state of Sergipe, were evaluated. The patients were divided into 7 groups according to the location at the onset of symptoms, following the existing division of health regions in the state. For comparison between groups, a significant difference was considered when $p<0.05$.

Results: Of the total of 844 patients suffering from STEMI who were transferred to the hospital with PPCI that serves SUS patients, 386 patients (45.8\%) underwent primary angioplasty. The mean rate of fibrinolytic use was $2.6 \%$, with no differences between the regions. The mean total time of arrival to the hospital with PPCI was 21 hours and 55 minutes, with a median of 10 hours and 22 minutes (6 hours and 30 minutes to 22 hours and 52 minutes). Total 30-day mortality was 12.8\%, but without differences between the regions, even when adjusted for age and sex.

Conclusions: This study reveals that fibrinolytics are underused throughout the state and that there is a significant delay in access to the hospital with PPCl, in all health regions of Sergipe.

Keywords: Cardiovascular Diseases; Myocardial Infarction; Myocardial Reperfusion; Mortality; Epidemiology; Cross-Sectional Studies

Full texts in English - http://www.arquivosonline.com.br

\section{Introdução}

As doenças cardiovasculares (DCV) representam a principal causa de morte no Brasil e no mundo. Dentro desse grupo, o infarto agudo do miocárdio com supradesnivelamento do segmento ST (IAMCSST) é responsável pela maior mortalidade na classe de doenças isquêmicas do coração, devido a sua severidade no prognóstico clínico. ${ }^{1}$

Nesse contexto, o acesso precoce e a imediata reperfusão coronariana é o principal objetivo no tratamento do IAMcSST, por reduzir os resultados adversos e mortalidade., ${ }^{2,3}$ Dentre as terapias de reperfusão a intervenção coronariana percutânea (ICP) primária e o uso de fibrinolítico são as principais estratégias terapêuticas. Entretanto a ICP, é considerada padrão ouro no tratamento, se realizada em menos de 12 horas do início dos sintomas e tem se mostrado superior ao fibrinolítico na redução da mortalidade, re-infarto e acidente vascular cerebral. ${ }^{4}$

Estudo prévio desenvolvido por Oliveira et al., ${ }^{5}$ mostrou que em Sergipe o tempo de chegada do início dos sintomas ao hospital com ICP $(24,4 \mathrm{~h} \pm 36,5 \mathrm{~h})$, é o dobro de tempo do preconizado aos pacientes do SUS. As taxas de uso de fibrinolítico apesar de serem baixas nos serviços público e particular, são ainda menores nos pacientes do SUS e a mortalidade em 30 dias foi maior no SUS $(11,9 \%)$ quando comparada aos pacientes do serviço privado (5,9\%). Esses dados podem ser ainda piores quando comparados entre as regiões de saúde de Sergipe. ${ }^{5}$

Ademais, baseado em princípios organizacionais do SUS, o estado é dividido em 7 regiões de saúde, porém, apesar da divisão, todos os hospitais com serviço de hemodinâmica estão situados em uma única região de saúde. E, para maior agravamento da situação, apenas um desses hospitais é referência cardiológica para os usuários do SUS e tem por característica não ser porta aberta.

Nesse contexto, o presente estudo objetiva avaliar as possíveis diferenças no tocante ao acesso às terapias de reperfusão e mortalidade em pacientes com IAMcSST atendidos exclusivamente pelo SUS entre as diferentes regiões de saúde de Sergipe.

\section{Materiais e métodos}

Trata-se de estudo transversal, com abordagem quantitativa, com dados obtidos de dezembro de 2014 a março de 2018 , que utilizou a base de dados do registro VICTIM (Via Crucis para Tratamento do Infarto do Miocárdio). Esta pesquisa foi aprovada pelo Comitê de Ética e Pesquisa da Universidade Federal de Sergipe, sob o número do parecer 483.749. Os dados foram coletados no único hospital do estado que conta com a disponibilidade de ICP primária pelo SUS. Este, por sua vez não possui sistema de porta-aberta, ou seja, os pacientes devem ser encaminhados de outros serviços de saúde já com o diagnóstico de IAMCSST confirmado.

A coleta foi realizada pelos pesquisadores mediante um instrumento próprio de pesquisa, o case report form (CRF), composto de variáveis sociodemográficas, condições clínicas à hospitalização, dados referentes ao tempo e caminho percorridos do início dos sintomas até o atendimento em hospital especializado, procedimento angiográfico e evolução dos pacientes durante a internação hospitalar após o IAM. As informações foram coletadas por meio de entrevista com o paciente ou acompanhante e dados do prontuário.

Foram incluídos pacientes de ambos os sexos, maiores de 18 anos, cujo início dos sintomas se deu dentro de território sergipano, com diagnóstico de IAMcSST confirmado pelo eletrocardiograma, de acordo com os critérios definidores propostos pela V Diretriz da Sociedade Brasileira de Cardiologia ${ }^{6}$, que tiveram atendimento fornecido exclusivamente pelo SUS e que assinaram o Termo de consentimento Livre e Esclarecido.

Utilizamos como critério de exclusão os indivíduos que: morreram antes da entrevista; que recusaram a participação na pesquisa; que tiveram início de sintomas fora de território sergipano; que receberam atendimento em rede privada; que não caracterizaram a Via Crucis, ou seja, aqueles pacientes que não percorreram o trajeto desde o início dos sintomas até a chegada ao hospital com capacidade de realizar ICP por terem apresentado o IAMCSST já dentro do hospital; aqueles cujo evento agudo de IAMCSST foi caracterizado como reinfarto (ocorreu dentro de 28 dias do infarto incidente); apresentaram mudança de diagnóstico durante a internação e os atendidos por convênio em hospital filantrópico. 
Obedecidos os critérios de inclusão do estudo, a alocação dos pacientes foi feita de forma consecutiva. Para a análise, os pacientes com IAMcSST foram divididos em 7 grupos, a partir da região de saúde de início de sintomas, são eles: 1. Aracaju, 2. Itabaiana, 3. Estância, 4. Lagarto, 5. Nossa Senhora do Socorro (Socorro), 6. Nossa Senhora da Glória (Glória), e 7. Propriá. Essas regiões de saúde foram delimitadas conforme a Deliberação no 065/2012, de 18 de abril de 2012, que ratifica a divisão do território estadual de Sergipe em 7 regiões de saúde, determinando os municípios que compõem cada região (Figura 1). O estado de Sergipe e seus 75 municípios, com base no último censo realizado pelo Instituto Brasileiro de Geografia e Estatística, possui população estimada pouco superior a dois milhões de habitantes, ${ }^{7}$ que se dividem, na perspectiva do SUS, nessas 7 regiões de saúde. ${ }^{2}$

\section{Análise Estatística}

As variáveis categóricas foram descritas por meio de frequência absoluta e relativa. As associações foram testadas por meio do teste qui-quadrado com simulações de MonteCarlo. As múltiplas comparações para as proporções foram testadas por meio de teste Z com correção de Bonferroni. As variáveis contínuas foram descritas por meio de mediana e intervalo interquartil devido a não aderência destas à distribuição normal avaliada pelo teste de Shapiro-Wilks. As diferenças nas medidas de tendência central foram testadas por meio do teste de Kruskal-Wallis. As múltiplas comparações para as medidas de tendência central foram testadas pelo teste de Kruskal-Wallis com correção de Bonferroni. Foram estimadas razões de chances brutas e ajustadas para a mortalidade geral em 30 dias por meio de regressão logística. O nível de significância adotado foi de 5\% e o software utilizado foi o R Core Team 2019.

\section{Resultados}

\section{Perfil Sociodemográfico}

Foram analisados 844 pacientes dos quais, 294 (34,8\%) eram da região de saúde Aracaju, 102 (12,1\%) da região Itabaiana, 119 (14,1\%) da região Estância, 122 (14,5\%) da região Lagarto, $119(14,1 \%)$ da região Socorro, 41 (4,85\%) da região Glória e 47 (5,6\%) da região Propriá.

A idade mediana total foi de 61 anos; dentre as regiões, Estância apresentou significativamente maior mediana de idade e a região Glória a menor. Em todas as regiões, houve prevalência do sexo masculino e da etnia não branca, havendo diferença entre a região Socorro quando comparada à Lagarto ou Glória ( $p=0,02)$ (Tabela 1).

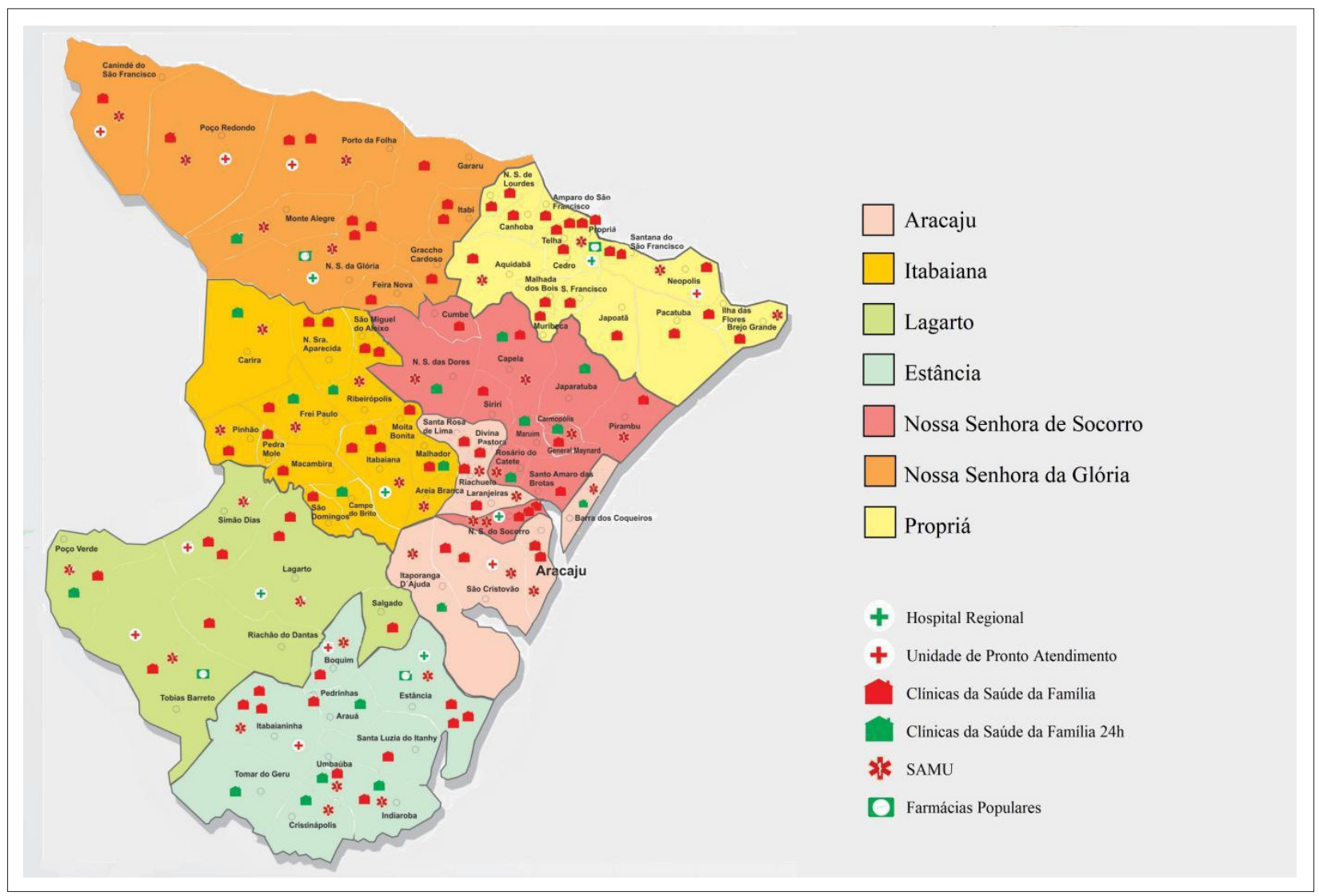

Figura 1 - Mapa de Sergipe e suas regiões de saúde. Fonte: (Secretaria De Estado de Saúde, 2016). 


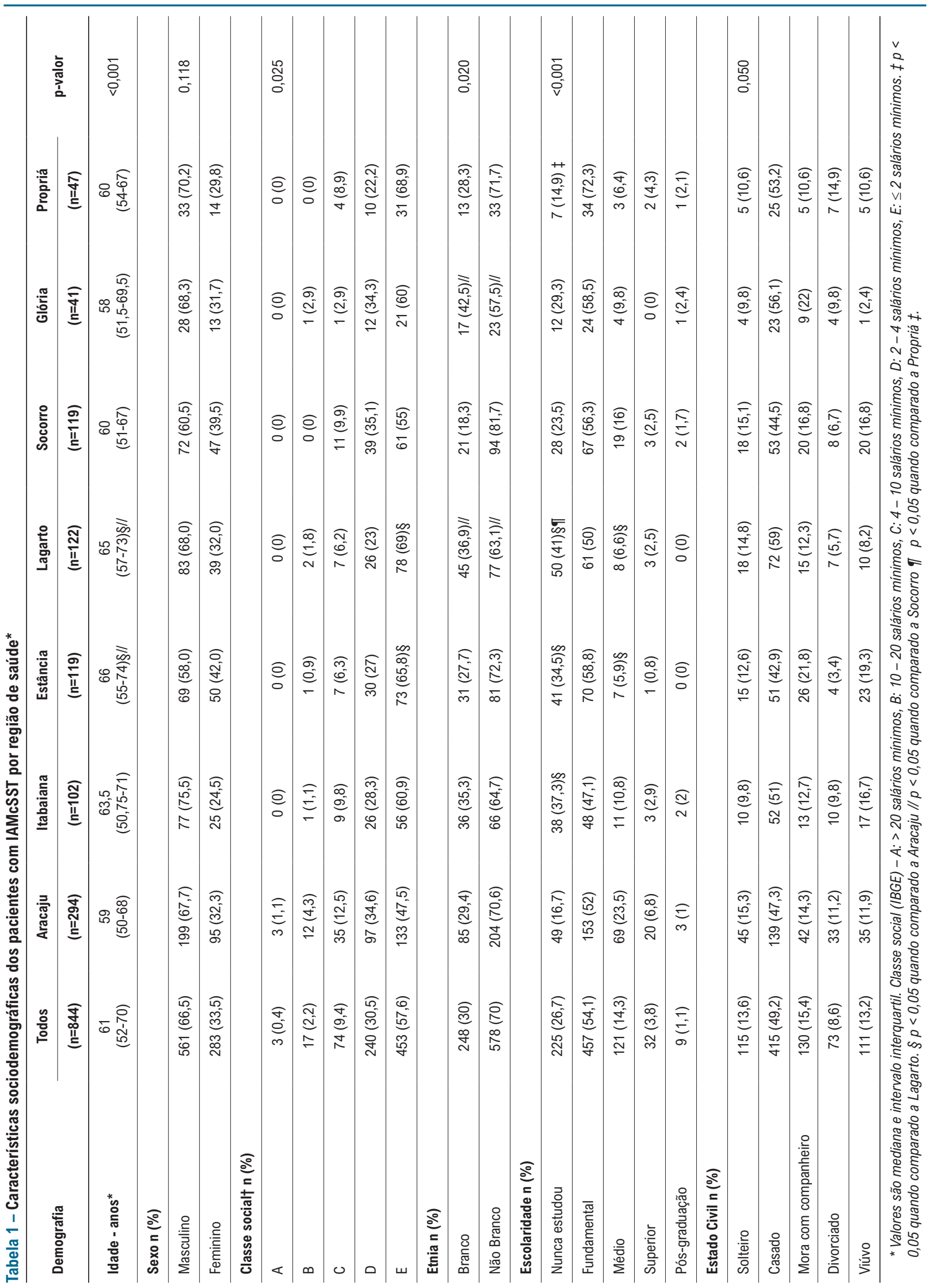




\section{Artigo Original}

\section{Características clínicas}

Dentre os fatores de risco, a diabetes mellitus foi a única que apresentou variabilidade significativa, oscilando entre $17,1 \%$ em Glória e 42,6\% em Propriá ( $p=0,026)$. Os demais fatores de risco apresentaram prevalências semelhantes entre os grupos (Tabela 2).

Quanto aos valores de pressão arterial sistêmica admissionais no hospital com ICP (Tabela 2), a pressão arterial sistólica teve mediana total de $140 \mathrm{mmHg}$, apresentando maior valor na região Aracaju e menor em Nossa Senhora da Glória, com diferenças quando comparadas entre si $(p=0,016)$. Já a pressão arterial diastólica, atingiu mediana total de $83 \mathrm{mmHg}$ apresentando maior mediana em Aracaju, Nossa Senhora do Socorro e Itabaiana e menor mediana em Nossa Senhora da Glória, Estância, Lagarto e Propriá ( $p=0,007$ ).

A região de Lagarto apresentou a maior taxa de pacientes considerados de alto risco de mortalidade pelo GRACE score (Tabela 2) (64,4\%), enquanto Aracaju apresentou a menor $(41,9 \%)$, sendo possível observar diferença quando comparadas as regiões de Aracaju e Nossa Senhora da Glória em relação à Lagarto $(p=0,001)$. Do total de pacientes, $85,3 \%$ apresentaram Killip I e 62,5\% dos IAMcSST foram de parede anterior. Esse padrão de comportamento se repetiu entre as regiões.

\section{Reperfusão coronariana}

A taxa de ICP primária total foi de $45,8 \%$, sendo a região Aracaju com a maior taxa (51,9\%) e Glória com a menor $(17,1 \%)$, notando-se diferença quando comparada à Aracaju ou Itabaiana $(p=0,03)$. Do total, $25,1 \%$ dos pacientes do estudo não realizaram ICP e a taxa de uso do fibrinolítico total foi de $2,6 \%$ (Tabela 3 ).

O tempo médio entre o início dos sintomas e a chegada em hospital com ICP foi de 21 h55' com mediana de 10h22' $\left(6 \mathrm{~h} 3 \mathrm{O}^{\prime}-22 \mathrm{~h} 52^{\prime}\right)$, sendo Glória a apresentar o maior atraso, e Aracaju o menor. Foi registrada diferença estatística quando comparadas regiões de Glória e Socorro em relação à Aracaju $(p=0,001)$. Dos períodos que compõem todo esse curso temporal, o tempo decorrido entre a chegada no hospital sem ICP até a chegada ao hospital com ICP foi o mais impactante, registrando um tempo mediano de 7 h37'. Nesse quesito, a região de maior atraso foi Clória, e a de menor Aracaju, sendo possível observar diferenças quando comparadas as regiões de Lagarto e Glória frente a Aracaju (Tabela 4).

Em relação ao número de instituições percorridas, até o hospital com ICP, a grande maioria dos pacientes $(81,2 \%)$ passaram por pelo menos uma instituição antes do hospital com ICP. Cerca de 2,4\% dos pacientes passaram por pelo menos três instituições antes do hospital com ICP, enquanto que apenas $1,7 \%$ tiveram acesso direto a este hospital (Tabela 4).

\section{Mortalidade}

Ao avaliar a mortalidade em 30 dias (Tabela 5), observouse que a região de Estância obteve a maior taxa (18,6\%) e Nossa Senhora da Glória a menor $(7,5 \%)(p=0,03)$. Quando ajustada para idade e sexo, adotando a região Aracaju como grupo controle, não foram observadas diferenças estatísticas.

\section{Discussão}

Três principais achados marcaram expressivamente os resultados desse estudo. O primeiro aponta grandes atrasos na chegada dos pacientes com IAMcSST no hospital com ICP, independentemente do local de início dos sintomas. O segundo atesta disparidades no uso das terapias de reperfusão entre pacientes nas diferentes regiões de saúde. O terceiro mostra a influência regional na mortalidade. Tais achados demonstram que há necessidade urgente de melhorarias na qualidade assistencial para pacientes com IAMcSST em todo o estado de Sergipe.

Apesar do maior conhecimento a respeito das metas terapêuticas no manejo do IAMcSST, a reprodutibilidade desses alvos ainda é tarefa difícil, principalmente no âmbito da saúde pública no Brasil. ${ }^{8,9}$ Os pacientes que iniciam o quadro de IAMcSST no estado de Sergipe possuem uma taxa de mortalidade em 30 dias longe do que se considera desejável. Um registro realizado pela Sociedade Europeia de Cardiologia, incluindo países, mostrou mortalidade intrahospitalar variando de 3,1\% a 6,1\%. ${ }^{10} \mathrm{Um}$ ensaio francês, executado entre os anos de 1995 a 2010, constatou uma queda na mortalidade em 30 dias de $13,7 \%$ para $4,4 \%$. Esse decréscimo se deve a múltiplos fatores dentro da assistência ao paciente com IAMcSST, como o aumento na quantidade de unidades de terapia intensiva móveis, aumento no número de campanhas públicas informativas a respeito dos sintomas relacionados ao agravo e menor atraso tanto na chegada em hospital capacitado, quanto na decisão de procurar socorro. ${ }^{11}$

Concomitantemente, ambas as regiões com piores taxas apresentavam as maiores faixas etárias médias. Apesar da redução da mortalidade por síndromes coronarianas agudas em todas as idades, sabe-se que pacientes com maior faixa etária apresentam pior prognóstico frente aos mais jovens, tanto por apresentarem maior número de comorbidades quanto por menor uso das terapias de reperfusão e medicamentosas. ${ }^{3,12,13}$

Antes de chegarem ao hospital com capacidade de realizar ICP, alguns pacientes necessitaram passar primeiramente por pelo menos uma unidade de saúde sem este serviço até serem transferidos para o hospital especializado. Esses dados também foram analisados neste estudo e obteve-se como resultado que a maioria dos pacientes passou por pelo um hospital antes de chegar ao hospital com capacidade para realizar ICP, fato este que já era esperado, uma vez que o único hospital público com capacidade de realizar ICP não possui atendimento de porta aberta. Alguns pacientes que tem acesso direto ao hospital com ICP foram regulados e encaminhados pelo SAMU.

O período entre o início dos sintomas e o acesso a um serviço de hemodinâmica apresenta papel decisivo no prognóstico do paciente. ${ }^{14} \mathrm{Em}$ Sergipe, nesse período o tempo dispendido foi em média de 21 horas e 55 minutos com mediana de $10 \mathrm{~h} 22^{\prime}$ ( $\left.6 \mathrm{~h} 3 \mathrm{O}^{\prime}-22 \mathrm{~h} 52^{\prime}\right)$, beirando o dobro da janela de 12 horas estabelecidas pelas diretrizes nacionais e internacionais. Avaliando numa perspectiva regional, esse intervalo médio alcançou valores de 26 horas e 24 minutos e 26 horas e 10 minutos nas regiões de Socorro e Glória, respectivamente, sendo mais curto em Estância, com 16 horas 


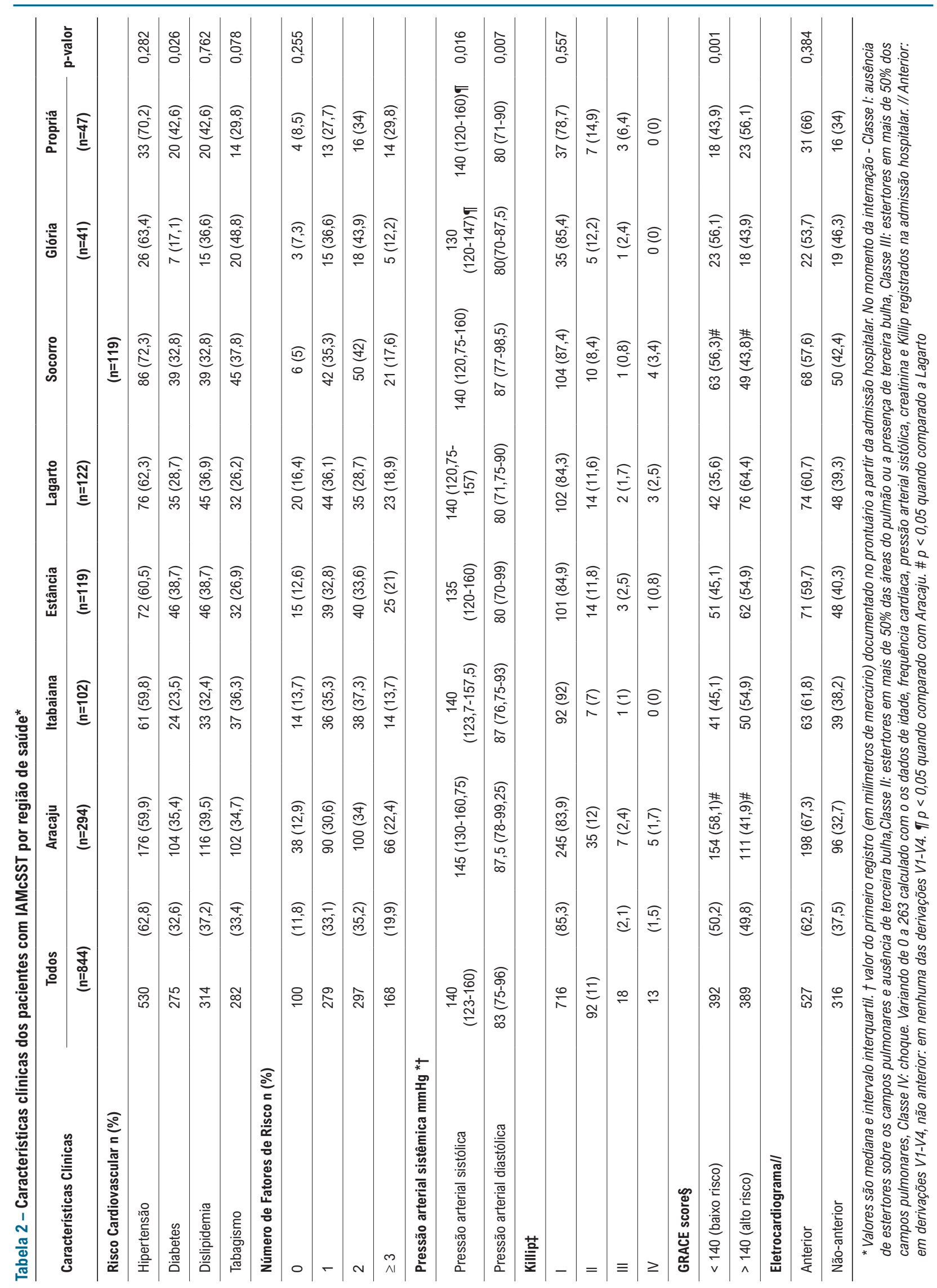




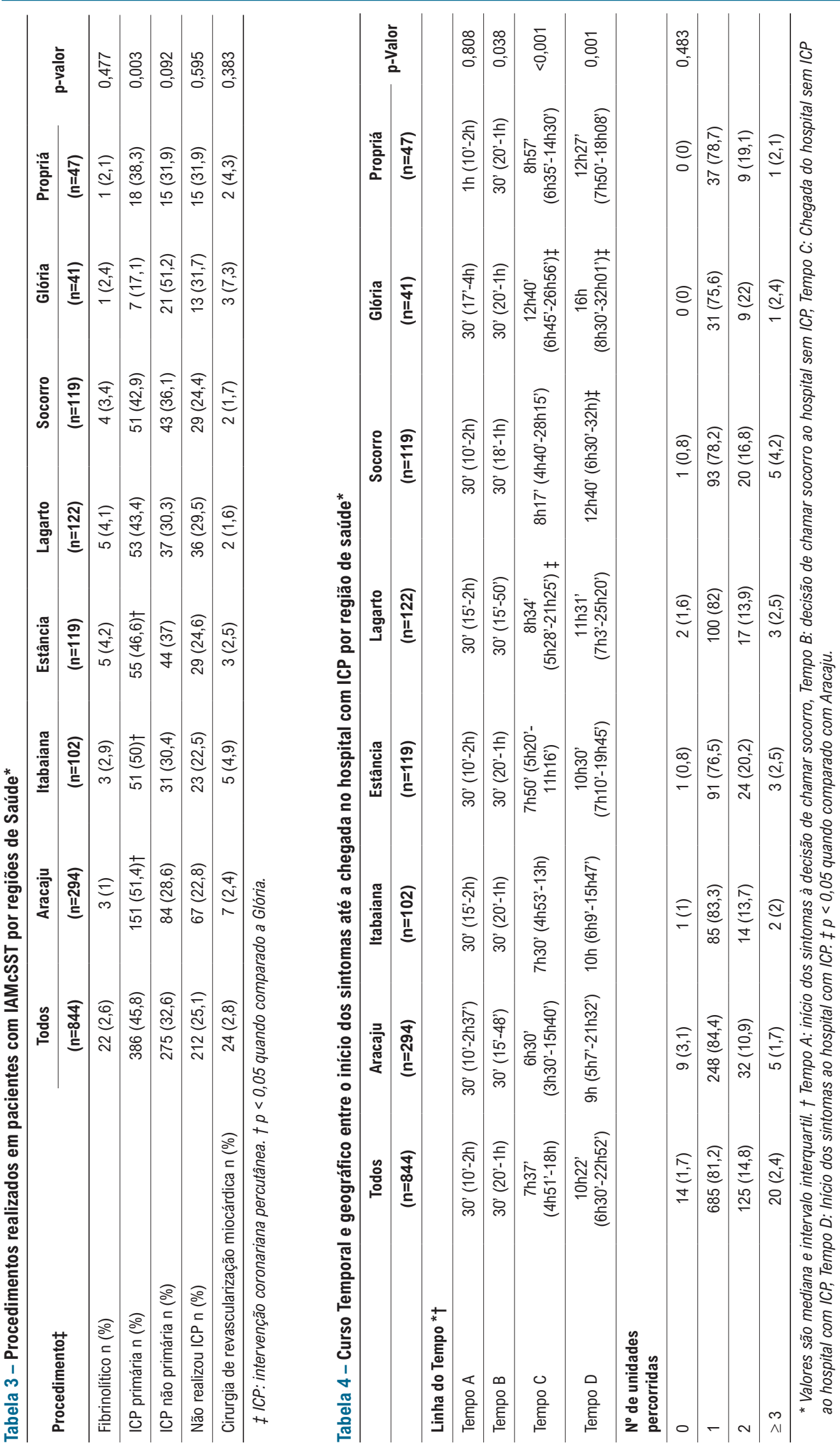


Tabela 5 - Razão de chances da mortalidade em 30 dias nos pacientes com IAMcSST por região de saúde ajustada por idade e sexo

\begin{tabular}{lcccc}
\hline Regiões de saúde & Mortalidade N (\%) & $\begin{array}{c}\text { Mortalidade não ajustada } \\
\text { OR (IC95\%) }\end{array}$ & Mortalidade ajustada OR (IC95\%) & p-valor \\
\hline Aracaju* & $27(9,4) 27(9,4)$ & 1 & 1 & $0,93(0,32-2,70)$ \\
\hline Itabaiana & $9(9,2)$ & $0,98(0,44-2,16)$ & $1,70(0,74-3,92)$ & 0,214 \\
\hline Estância & $22(18,6)$ & $2,22(1,20-4,08)$ & $2,07(0,93-4,61)$ & 0,074 \\
\hline Lagarto & $22(18,5)$ & $2,19(1,19-4,03)$ & $2,04(0,86-4,87)$ & 0,106 \\
\hline Socorro & $15(12,7)$ & $1,41(0,72-2,75)$ & $0,66(0,13-3,48)$ & 0,625 \\
\hline Glória & $3(7,5)$ & $0,78(0,23-4,68)$ & $1,93(0,61-6,11)$ & 0,263 \\
\hline Propriá & $8(17)$ & $1,98(0,84-4,68)$ &
\end{tabular}

*Representa o grupo controle.

e 22 minutos. No entanto, apesar dessa discrepância, só foi possível observar diferenças estatísticas quando comparadas Socorro e Glória à Aracaju. Vale ressaltar que o município mais distante da capital está a cerca de 3 horas de distância.

No estado de Sergipe, o curso temporal vivido por pacientes com IAMcSST desde o início dos sintomas até o acesso a hospital capacitado sofre enorme impacto do período inter-hospitalar, constituindo aproximadamente $87 \%$ de todo o processo. Nessa perspectiva, esses resultados não correspondem à realidade geográfica de Sergipe, que, considerado o menor estado da Federação, é necessário em torno de 4 horas de carro para ir de uma extremidade à outra do estado. Além disso, a região de Socorro, apesar da relativa proximidade à região Aracaju frente às demais, paradoxalmente, apresenta o maior desses hiatos com 23 horas e 15 minutos.

Vários elementos podem se atrelar ao prolongamento desse intervalo, podendo variar desde atrasos no diagnóstico da doença até ineficiência nos métodos de transporte entre as instituições assistentes. ${ }^{1,15}$ Um dado que merece destaque, é a impossibilidade de encaminhamento dos pacientes diretamente ao hospital habilitado. Em Sergipe, o único hospital capaz de ofertar tratamento definitivo para pacientes portadores de IAMcSST do SUS apenas acolhe pacientes mediante encaminhamento realizado por outra instituição, desde que já diagnosticados.

Um estudo realizado no Estado da Carolina do Norte, no período entre os anos de 2008 e 2010 avaliou 1.288 pacientes com diagnóstico de IAMcSST, dividindo-os em dois grupos; aqueles que eram transferidos diretamente para Hospitais com ICP independente da distância e aqueles que eram transportados para o hospital mais próximo sem ICP. Nessa comparação, 46,5\% dos pacientes transferidos diretamente para um centro com serviço de hemodinâmica chegavam dentro dos 90 minutos após primeiro contato médico, enquanto no outro grupo apenas $21,5 \%$ dos pacientes alcançavam um centro de hemodinâmica dentro dos 120 minutos após primeiro contato médico. ${ }^{16}$

Considerando-se a grande dificuldade de acesso no único hospital com capacidade de realizar ICP primária, menos da metade dos pacientes chegam na janela das $12 \mathrm{~h}$ do início dos sintomas, o expressivo subuso do fibrinolítico no estado de Sergipe pode ser considerado um indicador de péssima prática assistencial e contribuinte para a elevada mortalidade observada no nosso registro. A taxa média de uso de fibrinolítico de $2,6 \%$ difere bastante de outros registros ${ }^{17,18}$ nacionais e internacionais e, no cenário de dificuldade de acesso ao único hospital com capacidade de realização de ICP primária, expõe de forma inequívoca a grande fragilidade e ineficiência do atendimento do IAMcSST no nosso estado. ${ }^{4}$ Esses nossos achados poderiam ser até mais críticos, se a população estudada fosse composta de todos os pacientes com IAMcSST do estado, ou seja, os que ficaram em hospitais primários e secundários, sem acesso ao hospital terciário. Municiar a rede de hospitais regionais com capacidade de realizar trombólise no cenário poderá aumentar a taxa total de pacientes reperfundidos e reduzir a mortalidade nesse cenário.

Apesar de a angioplastia primária ser o tratamento de escolha para esses pacientes. No presente estudo, apenas 45,8\% dos pacientes foram submetidos a essa terapêutica, atingindo valores mais gritantes na perspectiva da região Glória, com $17,1 \%$. Estas taxas estão abaixo do que se encontra em outros estudos. ${ }^{19-22}$ Apesar da variabilidade entre as regiões, só foi possível observar diferença estatística quando comparada a região de Lagarto a Aracaju.

As discrepâncias encontradas tanto na taxa de ICP, quanto no tempo de acesso a esse método, se justificam pelas mesmas falhas da rede assistencial. Um estudo conduzido no Reino Unido, mostrou que, na segunda metade de 2011, 94\% dos pacientes com IAMcSST foram tratados por meio da ICP, aumento significativo quando comparado a taxa de $46 \%$ no ano de 2008. Dentre os elementos responsáveis nessa progressão estão: transporte de pacientes diretamente para centros com ICP, treinamento profissional para diagnóstico pré-hospitalar, coleta de dados a respeito da qualidade da assistência, e por último, criação de políticas nacionais no sentido de facilitar o acesso à rede de saúde. ${ }^{23}$

Um outro estudo realizado no Reino Unido utilizando dados da Inglaterra e do País de Gales, contando com um total de 228 hospitais e uma amostragem de 34.722 pacientes com IAMcSST, demonstrou que o uso de aspirina na admissão e a trombólise fora de ambiente hospitalar são os mais fortes preditores de sobrevivência intra-hospitalar. Além disso, fatores como frequência cardíaca e pressão arterial sistólica admissionais também impactam na mortalidade em 30 dias por IAMcSST. ${ }^{3}$ 


\section{Limitações}

Tendo em vista a precariedade de registros bem documentados em algumas das regiões, a obtenção dos dados foi complementada por meio de entrevista e parte destes foram auto referidos, o que pode possibilitar imprecisões nas medidas temporais. Também vale notificar que, muitos usuários do SUS desconhecem seu estado de saúde prévio, dificultando a mensuração precisa da prevalência das comorbidades, esse fato dificulta o ajuste de risco entre as diferentes populações das regiões investigadas. Por último, o presente estudo restringiu a coleta de dados ao hospital com ICP, por ser a única referência em tratamento de IAMcSST no estado. Esse fato limita os resultados aos pacientes que tiveram acesso ao centro de referência. Entretanto, destacamos que por limitarmos a população ao centro de referência, o cenário observado deve representar a melhor qualidade assistencial praticada pelo SUS no estado de Sergipe.

\section{Conclusão}

O registro VICTIM flagrou gritante hipossuficiência quanto ao acesso a ICP no estado, registrando uma janela temporal de acesso primário quase o dobro do que se considera limítrofe, sendo ainda pior em algumas sub-regiões. Ademais, existe uma subutilização marcante no uso dos fibrinolíticos, como terapia alternativa de reperfusão para o IAMcSST, em todas as regiões. Altas taxas de mortalidade persistem, apesar dos progressos terapêuticos da ciência cardiovascular na era da reperfusão miocárdica. Em conjunto, nossos dados demonstram uma grande ineficiência do SUS, no quesito assistência ao paciente com IAMcSST, no estado de Sergipe. Nossos resultados deveriam ser investigados em outros estados

\section{Referências}

1. Farshid A, Allada C, Chandrasekhar J, Marley P, McGill D, O'Connor S, et al.. Shorter ischaemic time and improved survival with pre-hospital STEMI diagnosis and direct transfer for primary PCI. Heart Lung Circ. $2015 ; 24(3): 234-40$.

2. França AVC.Atenção Hospitalar no Estado de Sergipe: Saberes e tecnologias para implantação de uma política. In:França AVC. Atenção Hospitalar no Estado de Sergipe. Sergipe: Sergipe:Fundação Estadual de Saúde;2021.p.1-188

3. Gale CP, Cattle BA, Woolston A, Baxter PD, West TH, Simms AD, et al. Resolving inequalities in care? Reduced mortality in the elderly after acute coronary syndromes. The Myocardial Ischaemia National Audit Project 2003-2010. Eur Heart J. 2011; 33(5):630-9.

4. Gershlick AH, Banning AP, Myat A, Verheugt WA, Gersh BJ. Reperfusion therapy for STEMI: is there still a role for thrombolysis in the era of primary percutaneous coronary intervention?. Lancet. 2013; 382(9892):624-32.

5. Oliveira JC, Santos MA, Oliveira JC, Oliveira LC, Barreto ID, Lima TC, et al. Disparities in Access and Mortality of Patients With ST-Segment elevation Myocardial Infarction Using the Brazilian Public Healthcare System: VICTIM Register. J Am HeartAssoc. 2019;8(20):e013057.

6. Piegas LS, Timerman A, Feitosa GS, Nicolau JC, Mattos LA, Andrade MD, et al. V Diretriz da Sociedade Brasileira de Cardiologia sobre Tratamento do Infarto Agudo do Miocárdio com Supradesnível do Segmento ST. Arq Bras Cardiol. 2015;105(2):1-105. e regiões do país para avaliar se os precários indicadores observados no presente estudo são peculiares de Sergipe, da região inserida ou representam o "padrão" do atendimento do sistema de saúde pública no Brasil.

\section{Contribuição dos autores}

Concepção e desenho da pesquisa e Revisão crítica do manuscrito quanto ao conteúdo intelectual importante: Oliveira JC, Ferreira GJS, Oliveira JC, Lima TCRM, Barreto IDC, Oliveira LCS, Arcelino LAM, Sousa AC, Barreto-Filho JAS; Obtenção de dados: Oliveira JC, Ferreira GJS, Oliveira JC, Lima TCRM, Barreto IDC, Oliveira LCS, Arcelino LAM; Análise e interpretação dos dados: Oliveira JC, Ferreira GJS, Oliveira JC, Lima TCRM, Barreto IDC, Arcelino LAM, Sousa AC, Barreto-Filho JAS; Análise estatística: Oliveira JC, Barreto IDC; Obtenção de financiamento: Oliveira JC, Oliveira JC, Lima TCRM, Oliveira LCS, Barreto-Filho JAS; Redação do manuscrito: Oliveira JC, Ferreira GJS, Barreto-Filho JAS.

\section{Potencial conflito de interesse}

Não há conflito com o presente artigo

\section{Fontes de financiamento}

O presente estudo não teve fontes de financiamento externas.

\section{Vinculação acadêmica}

Este artigo é parte de dissertação de mestrado de Jeferson Cunha Oliveira pela Universidade Federal de Sergipe.

7. InstitutoBrasileiro de Geografia e Estatística.(IBGE). Estimativa populacional 2018. Disponível em: <https://www.ibge.gov.br/ estatísticas-ovoportal/sociais/populacao/9103-estimativas-de-populacao. html $=\& \mathrm{t}=$ downloads $>$. Acesso em: 06 de fevereiro de 2018.

8. Carvalho G, Rassi S, Guerios N, Curado FA, Bastos AT. Striving to meet targets for ideal treatment of acute myocardial infarction in Brazil: Data from the Midwest region. J Interv Cardiol. 2018;31(4):450-4.

9. Piegas LS e Haddad N. Intervenção coronariana percutânea no Brasil: resultados do Sistema Único de Saúde. Arq Bras Cardiol. 2011;96(4):31724.

10. Kristensen SD, Laut KG, Fajadet J, Kaifoszova Z, Kala P, Mario C, et al. Reperfusion therapy for ST elevation acute myocardial infarction 2010/2011: current status in 37 ESC countries. Eur Heart J. 2014;35(29):1957-70.

11. Puymirat E, Simon T, Steg PG, Schiele F, Gueret P, Blanchard D, et al. Association of changes in clinical characteristics and management with improvement in survival among patients with ST-elevation myocardial infarction. JAMA. 2012;308(10):998-1006.

12. Halon DA, Adawi S, Dobrecky-Mery I, Lewis BS. Importance of increasing age on the presentation and outcome of acute coronary syndromes in elderly patients. J Am Coll Cardiol. 2004;43(3):346-52.

13. Tran CT, Laupacis A, Mamdani MM, Tu JV. Effect of age on the use of evidence-based therapies for acute myocardial infarction. Am Heart J. 2004;148(5):834-41. 
14. Blankenship JC, Skelding KA, Scott TD, Berger PB, Parise H, Brodie BR, et al. Predictors of reperfusion delay in patients with acute myocardial infarction undergoing primary percutaneous coronary intervention from the HORIZONS-AMI trial. Am J Cardiol. 2010;106(11):1527-33.

15. Postma S, Dambrink JH, Boer MJ, Gosselink AT, Ottervanger JP, Koopmans $\mathrm{PC}$, et al. The influence of residential distance on time to treatment in STelevation myocardial infarction patients. Neth Heart). 2014;22(11):513-19.

16. Fosbol EL, Granger CB, Jollis JG, Monk L, Lin L, Lytle BL, et al. The impact of a statewide pre-hospital STEMI strategy to bypass hospitals without percutaneous coronary intervention capability on treatment times. Circulation. 2013;127(5):604-12.

17. Rosselló X, Huo Y, Pocock S, Werf FV, Chin CT, Danchin N, et al. Global geographical variations in ST-segment elevation myocardial infarction management and post-discharge mortality. Int J Cardiol. 2017;(245):27-34.

18. Vora AN, Holmes DN, Rokos I, Roe MT, Granger CB, French WJ, et al. Fibrinolysis use among patients requiring interhospital transfer for STsegment elevation myocardial infarction care: a report from the US National Cardiovascular Data Registry. JAMA. 2015;175(2):207-15.

19. Aguirre FV, Varghese JJ, Kelley MP, Lam W, Lucore CL, Gill JB, et al. Rural interhospital transfer of ST-elevation myocardial infarction patients for percutaneous coronary revascularization: the Stat Heart Program. Circulation. 2008;117(9):1145-52.

20. Ibanez B, James S, Agewall S, Antunes MJ, Bucciarelli-Ducci C, Bueno H, et al. Task Force on the management of ST-segment elevation acute myocardial infarction of the European Society of Cardiology (ESC). ESC Guidelines for the management of acute myocardial infarction in patients presenting with ST-segment elevation. Eur Heart J. 2018;39(2):119-77.

21. O'Gara PT, Kushner FG, Ascheim DD, Casey DE Jr, Chung MK, de Lemos JA, et al. 2013 ACCF/AHA Guideline for the Management of ST Elevation Myocardial Infarction: A Report of the American College of Cardiology Foundation/American Heart Association Task Force on Practice Guidelines. Circulation. 2013;127(4):362-425.

22. Santos J, Meira KC, Camacho AR, Salvador PT, Guimaraes RM, Pierin AM, et al. Mortalidade por infarto agudo do miocárdio no Brasil e suas regiões geográficas: análise do efeito da idade-período-coorte. Cien Saud Colet. 2018;(23):1621-34

23. Mclenachan JM, Gray HH, Belder MA, Ludman PF, Cunningham D, Birkhead J. Developing primary $\mathrm{PCl}$ as a national reperfusion strategy for patients with ST-elevation myocardial infarction: the UK experience. Eurolntervention. 2012;(8):99-107. 